\title{
ELECCIÓN DE TEORÍA, JUICIOS DE VALOR Y RACIONALIDAD EN KUHN
}

\author{
Germán GUERRERO PINO \\ Escuela de Filosofia \\ Universidad del Valle
}

\section{Planteamiento del problema}

El proceso de elección de teoría, los juicios de valor que se encuentran involucrados en dicho proceso y el poder calificar de racional o no la decisión de un científico a la hora de optar por una teoría u otra, son temas estrechamente relacionados entre sí y con el problema más general de lograr una explicación del desarrollo científico acorde con lo que históricamente se ha dado en la actividad científica. Es claro que una mejor comprensión de estos tres asuntos contribuye a - más no agota el- entender el fenómeno más general del desarrollo científico; problema, este último, que ha ocupado de manera importante la actividad de los filósofos de la ciencia desde sus mismos orígenes inmediatos en el presente siglo y muy especialmente a partir de la década de los sesenta, cuando el historiador y filósofo de la ciencia T. Samuel Kuhn publicara su polémico libro La estructura de las revoluciones cientificas (1962), en donde se ocupa de este fenómeno general de la actividad científica y presenta tesis novedosas sobre los tres temas planteados.

De modo que considero instructivo profundizar en los planteamientos que Kuhn presenta al respecto, teniendo en consideración no sólo el hecho mencionado de las rupturas que produjo en la filosofía de la ciencia dominante en la primera mitad del presente siglo, sino que también, y lo que es más significativo, a sabiendas de que: primero, su punto de vista al respecto, inicialmente plasmado en La Estructura, sufrió modificaciones dignas de análisis y consideración; y, segundo, no se puede desconocer que esta propuesta original de Kuhn tuvo gran influencia en el desarrollo de la problemática, especialmente 
en algunos sociólogos de la ciencia que asumieron esta obra como una fuente importante de tesis y problemas para su campo, y a partir de la cual llegan algunos a afirmar que los procesos de evaluación y elección de teorías dependen exclusivamente de factores sociales y culturales, dejándose por fuera toda consideración sobre el mundo natural que se quiere conocer.

Cuando se habla de modificaciones en el punto de vista inicial de Kuhn, no se quiere dar a entender con ello que dichas modificaciones estén acompañadas de cambios radicales y sustanciales en sus tesis iniciales sobre la elección de teorías. Estos cambios pueden ser calificados mejor como "matizaciones" más que como «alteraciones sustanciales». Son matizaciones en dos sentidos: como búsqueda de precisión y rigor en los análisis y conceptos inicialmente presentados, y como búsqueda de solución a ciertos problemas -estoy pensando especialmente en el de la racionalidad en la elección de teoría- puestos por la interpretación dominante que no habían sido en modo alguno tratados en la propuesta inicial, pero a partir de la cual era posible derivar una respuesta a los mismos.

En otras palabras, el presente trabajo no pretende abordar la problemática de la elección de teoría y racionalidad en la ciencia en toda su complejidad sino que busca más bien contribuir al mismo centrándose en la evolución o el desarrollo que sufrió el pensamiento de Kuhn en este respecto. Encuentro que es posible determinar tres etapas en la transformación del punto de vista de Kuhn, que ordenadas temporalmente coinciden, de manera importante, con los escritos donde Kuhn trata directamente el tema: La Estructura de las revoluciones cientificas (1962); Objetividad, juicios de valor y elección de teoria (1977) '; Racionalidad y elección de teoría (1983). Kuhn también habla al respecto en el Postscrip ${ }^{2}$ de 1969 a la segunda edición de La Estructura de las revoluciones científicas, pero considero que las diferencias que se pueden presentar en el Postscrip en relación con Objetividad, juicios de valor y elección de teoría radican más bien en aspectos de presentación: Kuhn en la Postscrip adelanta muchas de las tesis que desarrollará de manera más clara y sistemática en su

1 Si bien este artículo se publicó por primera vez en este año como parte del libro La Tensión Esencial, el mismo recoge la conferencia Machette pronunciada por Kuhn en la Furman University el 30 de noviembre de 1973.

2 A esta etapa también pertenecen los escritos Consideración en torno a mis críticos (1970) y Notas sobre Lakatos (1971), en donde Kuhn reitera muchas de las tesis principales sobre la elección racional de teoría. 
posterior escrito y no se encuentran diferencias conceptuales de fondo en el tratamiento del tema.

Con el objeto de poder caracterizar de manera general estas tres etapas y, con ello, mostrar en líneas generales las ideas básicas que el presente escrito quiere defender, es preciso decir algo sobre la línea directriz que permite visualizar la transformación que se propone. Dicha línea directriz tiene que ver, como es natural, con la elección de teoría, los criterios de elección y la racionalidad en la ciencia, y queda precisada en las siguientes pr000eguntas:

a) ¿Qué importancia otorga Kuhn a los factores internos y externos, respectivamente, en el proceso de elección de teoría?

b) De acuerdo con la caracterización de los modelos racional y no racional del cambio científico, que se hará en el siguiente numeral, qué tipo de modelo está proponiendo Kuhn?

c) ¿Qué dice Kuhn respecto a la racionalidad de la actividad científica; es decir, de la racionalidad en la elección entre teorías?

Estas cuestiones nos permiten, así mismo, caracterizar de modo general estas tres etapas, en los siguientes términos. En la primera etapa, la elección entre teorías kuhn la entiende como un proceso que se inicia con la persuasión y termina en la conversión del hombre de ciencia al nuevo paradigma, en donde si bien es posible esgrimir ciertos criterios internos de elección, que verdaderamente funcionan como valores, su eficacia es prácticamente nula dada la inconmensurabilidad presente entre los paradigmas en competencia y el hecho de que los mismos criterios o valores son uno de los elementos que permiten dar unidad a un paradigma. En la segunda etapa Kuhn presenta una tesis menos radical, puesto que la función de los criterios o valores de elección ya no es secundaria, como inicialmente lo pensaba, sino que de alguna forma influyen en, más no determinan, la elección de una teoría. Además, si bien Kuhn sigue manteniendo que "la transferencia de la fidelidad de un individuo, de una teoría a otra, sería mejor descrita como conversión y no como elección" ${ }^{3}$, el caso es que la dependencia de dichos criterios respecto a un paradigma - teoría - se hace ínfima, reduciéndose así las dificultades que la

3 KUHN, T. S., "Objetividad, juicios de valor y elección de teoria". En La tensión esencial, Fondo de Cultura Económica, México, 1997, p. 362. 
inconmensurabilidad de teorías inyectaba a la elección de teoría. En la última etapa se observa un movimiento más que precisa y consolida el punto de llegada anterior sobre los criterios de elección y en donde Kuhn aborda y presenta una solución al problema de la justificación de las normas para la elección racional de teorías, aproximando un concepto de ciencia a través de los criterios de elección y un concepto de racionalidad, noción que a lo largo de su trabajo anterior había eludido por parecerle enteramente a priori como la entendían sus adversarios ${ }^{4}$.

\section{Primera etapa}

Es necesario comenzar presentando, así sea de manera panorámica, las interpretaciones más dominantes en relación con el desarrollo o cambio científico. Por tanto, recurriremos a la presentación que hace Newton-Smith ${ }^{5}$ de los dos modelos dominantes y extremos que son posibles y que, de una u otra forma, han sido y siguen siendo defendidos por unos y otros profesionales en el campo del estudio de la ciencia. En otras palabras, cualquier propuesta teórica sobre la manera como cambia la ciencia es susceptible de ser involucrada, en líneas generales, en los modelos racionales de cambio científico o en los modelos no racionales o irracionales.

Un modelo racional comprende dos ingredientes: la meta de la ciencia y los principios que permiten lograr dicha meta. Es decir, en primer lugar, un modelo racional estipula algo como finalidad o meta de la ciencia; por ejemplo, la "búsqueda de la verdad», máxima que podemos considerar una herencia legada por los griegos y que con todo y ello puede ser objeto de escrutinio. Otros, como Popper, complementan esta máxima inicial al plantear que el objetivo de los científicos es producir teorías explicativas verdaderas. En tanto Kuhn arriesga una nueva finalidad: «la resolución de enigmas», en donde, por lo menos, la referencia a la verdad no se hace explícita. En segundo lugar, un modelo racional enuncia algún principio o conjunto de principios que permitan a los científicos

4 Ver por ejemplo: KUHN, T.S., "Consideración en torno a mis críticos», en La Critica y el Desarrollo del Conocimiento, Grijalbo, Barcelona, 1975, p. 432; y KUHN, T.S., "Notas sobre Lakatos", en La Crítica y el Desarrollo del Conocimiento, Grijalbo, Barcelona, 1975, pp. 514 y 520.

5 Newton-Smith, W. H., La racionalidad cientifica, Ediciones Paidós Ibérica, S. A., Barcelona, 1981. Para el punto que nos ocupa, ver especialmente el capítulo 1. La imagen racional. 
elegir entre teorías en competencia. Estos principios son lo que tradicionalmente se han llamado criterios de elección o evaluación de teorías, y que -como veremos más adelante, en la medida en que Kuhn es uno de los promotores de dicha ruptura- aparecen como valores epistemológicos, virtudes epistemológicas, desiderata o normas bajo la literatura de la metodología de la ciencia actual, con el propósito de destacar cierta ruptura con los modelos racionales.

Más aún, el modelo racional propone que estos principios que operan en el proceso de cambio científico son de carácter interno a la actividad científica; lo que es lo mismo, la elección de teoría se produce única y exclusivamente bajo la influencia de factores internos. En tanto que un modelo de explicación en sentido contrario al racional, un modelo no racional del cambio científico, privilegia exclusivamente los factores externos. "El adjetivo "interno" - plantea Newton-Smith - significa que los factores citados sólo se refieren a características de las teorías en cuestión y a circunstancias relativas a la relación entre las teorías y la evidencia disponible. Por el contrario, llamaremos factores externos a los factores psicológicos o sociológicos que no guardan relación con las teorías y la evidencia, sino con sus proponentes" ${ }^{6}$; es decir, factores relacionados con la capacidad propagandista, en donde los científicos están muy interesados en maximizar su prestigio en el seno de la comunidad científica, y el contexto social en el cual surge la teoría.

En la metodología de las ciencias se han presentado diversos candidatos para asumir la función de juez en las controversias entre teorías. Esto quiere decir que entre dos teorías rivales se debe elegir aquella que sea más:

1. Precisa: permite predecir con más precisión fenómenos pertenecientes al ámbito problemático.

2. Fecunda: resuelve viejos problemas y predice fenómenos totalmente insospechados desde otras alternativas teóricas.

3. Coherente: no es contradictoria internamente $y$, externamente, con teorías bien establecidas.

4. Amplia: reduce lo desordenado a lo más integrado; es decir, que integra dominios que anteriormente se presentaban como disímiles.

6 Ibid., p. 16. 
5. Simple: reduce lo relativamente complejo a lo más simple, se dice que la teoría es "más neta", "más apropiada" o "más sencilla" que otras alternativas teóricas 7 .

6. Nómica: reduce lo anómico a lo más nómico.

7. Verdadera: proporciona información sustancial verdadera sobre el ámbito en cuestión.

8. Explicativa (el argumento de la inferencia a favor de la mejor explicación): ofrece la mejor explicación hasta hora contemplada del campo problemático, a través de apropiadas predicciones en el dominio en cuestión, lo cual no había sido posible sin ella.

Una vez se ha precisado los dos modelos en disputa sobre el cambio científico, podemos pasar a enunciar lo que considero es la tesis más importante de Kuhn en lo relacionado con la elección de teoría. Tesis que se ha mantenido invariable en sus líneas generales en las sucesivas reflexiones kuhnianas respecto al tema y que es básicamente una crítica frontal a una de las ideas fundamentales defendida por el modelo racional. En pocas palabras, la tesis lo que propone es que los criterios de elección no sean vistos como reglas sino como valores; es decir, que realmente dichos criterios no operan como reglas de aplicación mecánicas que operan a manera de algoritmos que imponen y determinan de manera rígida la elección de una teoría, sino que por el contrario dichos criterios verdaderamente se emplean como valores que influyen en la decisión. En palabras de Kuhn: «la cuestión de la elección de paradigmas no puede resolverse nunca de manera inequívoca sólo mediante la lógica y la experimentación" ${ }^{8}$, "no hay un algoritmo neutral para la elección de teorías, no hay ningún procedimiento sistemático de decisión que, aplicado adecuadamente, deba conducir a cada individuo del grupo a la misma decisión" ${ }^{9}$.

7 La enunciación de estos cinco primeros criterios, si bien puede considerarse estándar, está inspirada en Kuhn. KuHN, T. S., "Objetividad, juicios de valor y elección de teoría”, p. 345.

8 Kunn, T. S., La estructura de las revoluciones cientificas, Fondo de Cultura Económica, México, 1983, p.153. En "Notas sobre Lakatos", análogamente plantea: "la elección entre paradigmas (o teorías, para lo que nos concierne ahora) no puede venir obligada sólo por la lógica y la experimentación; en estas cuestiones nada hay que sea demostración, ningún punto en el que quien se opone a una visión más nueva viole una regla de la ciencia, empiece a conducirse de una manera no científica", p. 521.

9 KuHN, T. S., La estructura de las revoluciones cientificas p. 304. En "Objetividad..." Kuhn dice (p. 355): "lo que estoy sugiriendo es que los criterios de elección con los que 
Las objeciones básicas de Kuhn a la tesis que asume los criterios como verdaderas reglas que dictan la elección entre teorías son las siguientes. Primera, de ser cierta la tesis debería darse el caso de que todos los científicos implicados en una crisis y ante la presencia de una teoría alterna tomaran la misma decisión: o bien mantenerse en la antigua teoría o bien comenzar a trabajar en la nueva teoría; pero tal cosa no sucede, históricamente nos encontramos con que hay científicos trabajando en ambos bandos. Segunda, la tesis presupone la invarianza interpretativa de cada criterio, pero lo cierto es que la manera de interpretar un mismo criterio, por ejemplo la amplitud, puede variar de manera asombrosa de un científico a otro. Tercera, la tesis igualmente presupone una ponderación constante de los criterios asumidos en su conjunto, pero este parámetro también puede variar de manera drástica de una comunidad científica a otra. Cuarta, contrariamente a lo supuesto por la tesis, la manera de entender los criterios puede cambiar a través de la historia y, quinta, la manera de entender un criterio también puede cambiar de un campo de aplicación a otro. En definitiva: no es posible establecer una fórmula matemática que contenga los diferentes criterios para poder hacer la elección.

Una vez aceptada la crítica fundamental de Kuhn a un modelo racional de elección de teoría, no hay problema alguno en emplear indistintamente términos como "criterio", "norma", "valor epistemológico", "virtud epistemológica" o "desiderata", dando por sobreentendido que dichos principios no son reglas que determinan de manera unívoca la decisión a tomar sino más bien algo que influye en la decisión.

Ahora bien, y teniendo en cuenta el objetivo de presentar las tres etapas por las que pasa Kuhn respecto a la elección de teorías, se dijo que la anterior tesis está presente en las tres etapas; esto es correcto, pero es necesario hacer cierta matización. Lo que se mantiene estrictamente en las tres etapas es el rechazo a entender los criterios como reglas mecánicas; pero la segunda parte de la tesis, aquella que plantea que los criterios influyen en la elección, es característica - como a continuación se mostrará- de la segunda etapa y no propiamente de la primera. Veamos esto último en detalle.

Para comenzar, hay que decir que Kuhn no fue lo suficientemente coherente a la hora de plantear la tesis que pretendía defender sobre el tema de

comencé funcionan no como reglas, que determinan decisiones a tomar, sino como valores, que influyen en éstas». 
elección de teorías, que es el que aquí nos interesa. En algunos casos está dispuesto a defender afirmaciones del siguiente tenor: los debates entre paradigmas «involucran siempre la pregunta: ¿Qué problema es más significativo resolver? Como la cuestión de la competencia de normas, esta cuestión de valores sólo puede contestarse en términos de criterios que se encuentran absolutamente fuera de la ciencia normal y es ese recurso a criterios externos lo que de manera más obvia hace revolucionarios los debates paradigmáticos» ${ }^{10}$; y en otras ocasiones, unas páginas más adelante, hace afirmaciones del siguiente tenor que difícilmente pueden conciliarse con la anterior, pues destacan la importancia de las virtudes epistémicas de una teoría en la evaluación: tipos de argumentos - plantea Kuhn - que resultan particularmente efectivos en las batallas sobre cambios de paradigmas: los partidarios del nuevo paradigma pueden resolver los problemas que condujeron al paradigma antiguo a la crisis; el nuevo paradigma permite la predicción de fenómenos totalmente insospechados cuando prevalecía el paradigma anterior; un llamamiento al sentido que tienen los individuos de lo apropiado y de lo estético: se dice que la nueva teoría es "más neta", "más apropiada" $o$ "más sencilla» que la antigua ${ }^{11}$.

Teniendo en cuenta deficiencias como la anterior en los argumentos que Kuhn presenta, y con el propósito de sacar adelante, bajo un mínimo de coherencia posible, las tesis fundamentales que Kuhn busca desarrollar en su libro La Estructura, hay que concluir que sus afirmaciones y argumentos más determinantes apuntan en la primera dirección, hacia aquella tesis en donde la elección entre paradigmas no puede estar determinada por factores internos sino por los estrictamente externos ${ }^{12} \mathrm{o}$, de manera más relajada, que por lo menos la función de los primeros puede ser entendida como secundaria.

Para complementar la idea de que los criterios se encuentran en un segundo plano respecto a los factores externos, sólo basta ver cómo Kuhn hace depender los valores del paradigma que se defiende; es decir, los criterios de elección varían categóricamente de un paradigma a otro o, lo que es lo mismo,

10 Ibid., p. 175.

11 Ibid., pp. 237-244.

12 NeWTON-SMITH plantea al respecto: «es posible que Kuhn haya llegado a su posición no racionalista a través de una hiperreacción a su afirmación tan sólida y repetida como tópica, de que no hay algoritmo para la elección de teorías rivales". NEWTON-SMITH, W. H., La racionalidad cientifica, p. 131. En "Consideración en torno a mis críticos" (pp. 404 y 419), Kuhn reitera que su enfoque en este sentido es claramente sociológico. 
de una teoría a otra. Esto es claro, puesto que su mismo concepto de paradigma, posteriormente precisado en el Postscrip a través del de "matriz disciplinar", involucra los valores como una de sus componentes; los restantes elementos de la matriz disciplinar son: las generalizaciones simbólicas, los ejemplares o paradigmas propiamente dichos y los paradigmas metafísicos ${ }^{13}$. De modo que al variar los valores de un paradigma a otro y dada la inconmensurabilidad entre estos, los valores mismos estarían sometidos a una suerte de inconmensurabilidad en donde la disputa entre paradigmas tiene que ser resuelta por factores externos ya que no es posible recurrir a criterios de elección neutrales. Kuhn en La Estructura dice: «La elección entre paradigmas en competencia resulta una elección entre modos incompatibles de vida de una comunidad...Cuando los paradigmas entran, como deben, en un debate sobre elección de un paradigma, su función es necesariamente circular. Para argüir en la defensa de ese paradigma cada grupo utiliza su propio paradigma» ${ }^{14}$.

¿Cómo entonces, desde el punto de vista de Kuhn, se produce la transferencia por parte de un científico de un paradigma a otro? En pocas palabras, a través de dos etapas: la persuasión y la conversión. "La transferencia de la aceptación de un paradigma a otro - plantea Kuhn - es una experiencia de conversión que no se puede forzar» ${ }^{15}$. Estas afirmaciones de Kuhn son muy problemáticas a la luz de la tesis de la inconmensurabilidad, que impediría cualquier tipo de diálogo entre los partidarios de paradigmas diferentes, y por tanto se diría que los factores internos no juegan papel alguno en la solución del conflicto de paradigmas. En La Estructura no hay una argumentación sistemática que permita precisar estos dos procesos, veremos que en la segunda etapa estos dos procesos se hacen más razonables dado que los criterios de elección son

13 El concepto de paradigma en Kuhn lo trato de manera más sistemática en "Inconmensurabilidad y Comparabilidad en Kuhn", en Thomas Kuhn, Editorial Universidad del Valle, Santiago de Cali, 1997, pp. 73-97; y en "Kuhn y el problema de la elección racional de teoría", en Falguera, J. L. y OTROS (Eds.), Proceedings of the Congress The Analytic Philosophy at the Turn of Millennium, pp. 343-353. Este último escrito y el presente se complementan, puesto que en el primero trato más las tesis metodológicas y epistemológicas que sustentan la tesis principal de la racionalidad de elección de teoría de Kuhn y propongo una jerarquía en los valores epistémicos, en tanto que el presente posee un carácter más histórico y menos crítico.

14 KuHN, T. S., La estructura de las revoluciones científicas, p. 152.

15 Ibid. p. 235. Y unas líneas más adelante (p. 236): “Cómo se induce a la conversión y cómo se encuentra resistencia? Tan sólo debido a que se refiere a técnicas de persuasión o a argumentos y contraargumentos en una situación en la que no puede haber pruebas». 
compartidos por la comunidad científica más amplia y el holismo semántico, asociado con la inconmensurabilidad, pasa de ser total a ser local.

\section{Segunda etapa}

En esta segunda etapa Kuhn presenta una tesis menos radical, puesto que la función de los criterios de elección, que verdaderamente funcionan como valores, ya no es secundaria como inicialmente lo pensaba sino que de alguna forma influyen en la elección entre teorías, más no la determinan. La tesis que aquí se está presentando la comparte igualmente Newton-Smith, pues dice: «el propio Kuhn ha expresado su acuerdo en los últimos tiempos, a saber, la del consenso con independencia de los paradigmas en lo relativo a las características que hacen de una teoría una buena teoría» ${ }^{16}$. En muchos lugares Kuhn expresa su distanciamiento de su tesis radical inicial, veamos algunos pasajes. En el Postscrip, al referirse a los valores como a una de las componentes de la matriz disciplinar plantea que: "habitualmente se les comparte entre diferentes comunidades...y hacen mucho para dar un sentido de comunidad a los científicos naturalistas en conjunto... Considero ahora como una flaqueza de mi texto original el haber prestado poca atención a valores tales como la coherencia interna y externa al considerar las causas de crisis y factores de elección de teorías» ${ }^{17}$.

Así pues, en este punto hay que ver cómo armoniza Kuhn la idea de un grupo de criterios compartidos con la idea de la inconmensurabilidad - local - entre teorías. Esta dificultad puede expresarse también del modo siguiente: ¿cómo es posible la comunicación entre miembros que hacen parte de teorías inconmensurables y, en últimas, cómo es posible la persuasión de un científico hacia un nuevo paradigma? Considero que Kuhn logra conciliar ambas ideas al poner de manifiesto que en el proceso de elección de una teoría intervienen dos momentos perfectamente distinguibles: la persuasión y la conversión.

De modo que en este punto Kuhn propone tratar a los científicos como miembros de comunidades lingüísticas diferentes y resolver el problema de la

16 NeWTON-SMITH, W. H., La racionalidad cientifica, p. 129.

17 KUHN, T. S., La estructura de las revoluciones científicas, p. 283. 
persuasión y de la conversión en términos de traducción entre dos lenguajes. Introduce, entonces, la distinción entre traducción y aprendizaje de un lenguaje: la traducción de manera parcial es la que permite persuadir a un individuo de las bondades de la nueva teoría mientras que la conversión requiere del aprendizaje del nuevo lenguaje y no es posible que se presente a la altura de la traducción parcial.

En forma más detallada, si partimos de que la inconmensurabilidad entre teorías, en términos generales, es divergencia de significados y, por otra parte, de que esta inconmensurabilidad es local dado que los miembros de comunidades enfrentadas comparten una buena porción de lenguaje y básicamente discrepan en cuanto al significado de los términos básicos de las teorías que defienden, bajo estas condiciones — plantea Kuhn - no es extra(o que sea posible una comunicación así sea a medias.

Bajo estas consideraciones, entonces, la aproximación del científico a la nueva teoría se inicia proponiendo traducciones parciales de los enunciados de la nueva teoría en términos de la vieja teoría acerca de experiencias que ambos científicos comparten, de tal manera que el proceso de transferencia de un científico a una nueva teoría se inicia precisamente porque el científico detecta, en primer lugar, que en ciertos aspectos mediante la implementación de la nueva teoría es posible un mayor acuerdo con la experiencia en comparación con la que actualmente práctica y, en segundo lugar, percibe que la nueva teoría logra dar respuesta a fenómenos antes no explicados como a nuevos fenómenos. En estas circunstancias el partidario del antiguo paradigma bien podría decir, como comenta el mismo Kuhn: "no se cómo lo lograron los partidarios de la nueva opinión, pero yo debo aprenderlo; sea lo que fuere lo que están haciendo, claramente tienen razón" ${ }^{18}$.

A esta altura el científico que practica la vieja teoría se encuentra persuadido por la nueva teoría, pero aún no ha hecho elección alguna. Este proceso culmina con la conversión del hombre de ciencia al nuevo paradigma o teoría y esto se logra a través de las sucesivas traducciones que hace el científico de la nueva teoría en términos de la vieja hasta que llega un momento en que deja de traducir y simplemente la aplica porque ha logrado comprender y entender los nuevos significados. La conversión es aprender el

18 Ibid., p. 309. 
nuevo lenguaje y esto se da de manera repentina en el proceso de aprender a traducir.

Para finalizar esta segunda etapa y con el propósito de trazar el puente con la siguiente, debemos plantearnos el problema de la justificación de los criterios de elección racional de teorías. Problema central desde una perspectiva enteramente filosófica de la ciencia y al cual no se han aproximado respuestas plausibles que permitan una mejor aproximación teórica al desarrollo de la ciencia. Es importante aclarar que Kuhn eludió, en cierta forma, en sus comienzos este problema y que por lo tanto fue renuente a emplear términos como "racional", «objetivo" y "verdad" en sus análisis, dado que los consideraba seriamente problemáticos y necesarios de una elaboración más sistemática de la que comúnmente daban de ellos, especialmente sus adversarios que defendían un modelo racional de la ciencia y que calificaban su enfoque como «irracional». En Objetividad, juicios de valor y elección de teoría, al tratar el tema sobre la objetividad y subjetividad de los valores, Kuhn es consciente de que su análisis no adelanta nada en relación con la justificación de los valores, pues comenta: «aunque la experiencia de los científicos no justifica filosóficamente los valores que sustentan - tal justificación resolvería el problema de la inducción--, tales valores se han aprendido en parte de la experiencia y han evolucionado con la misma" ${ }^{19}$.

En pocas palabras, la dificultad ante la cual se haya el enfoque de Kuhn y que Newton-Smith describe perfectamente es la siguiente: «aun cuando Kuhn retroceda en relación con su primera tesis fuerte acerca de la inconmensurabilidad como fruto de la variación radical del significado, y aun cuando acepte la existencia de convenios acerca de los principios de comparación que se utilizan en todos los paradigmas, aun así, digo, queda todavía mucha discrepancia entre él y el racionalista, cuya posición exige la justificación de los principios de comparación» ${ }^{20}$. Considero que Kuhn en su artículo Elección de teorías y racionalidad -que constituye lo que he llamado la tercera etapa de Kuhn en los planteamientos sobre la elección de teoría- aproxima una propuesta, la primera y única - hasta donde tengo entendido-, sobre la justificación de los criterios de elección racional de teorías.

19 KUHN, T. S., «Objetividad, juicios de valor y elección de teoría», p. 359.

20 NEWTON-SMITH, W. H., La racionalidad cientifica, p. 129. En la misma dirección, y refiriéndose a los planteamientos de Kuhn, dice Newton-Smith: «Si las reglas carecen de justificación, el mero hecho de emplearlas sólo significa que las decisiones se adoptan de acuerdo con un modelo previo, pero no que las decisiones tengan fundamentor. p. 131. 


\section{Tercera etapa}

Lo que conforma este numeral es, entonces, el desarrollo de las principales tesis de Kuhn en su último artículo mencionado y que tiene que ver, como también ya se ha dicho, con proponer una justificación de los criterios de elección, fundamentarlos teóricamente. En otras palabras, el principal problema del que se ocupa Kuhn en este escrito es el de presentar una justificación satisfactoria del empleo de ciertos criterios de elección de teoría y el de justificar en qué términos se podría decir que tal elección de teoría es racional o irracional.

El proceso de argumentación es el siguiente. Primero, se avanza la tesis según la cual, y bajo el principio metodológico de simplicidad, es más conveniente asumir los desiderata o criterios de elección como los objetivos o metas de la actividad científica y no ya como los principios que permiten lograr las metas - sobre este punto no se avanzará nada más-. El segundo paso tiene que ver con una digresión sobre la dicotomía normativo/descriptivo, que por tanto encierra más bien una reflexión sobre un aspecto metodológico determinante de la filosofía, pero que es vital para la argumentación que sigue. En este punto la posición final de Kuhn es que dicha disyuntiva no cabe, por lo menos, en el tema que se trata. Tercero, con vista a la primera parte del problema de la justificación de los criterios de elección, y haciendo uso de su enfoque sobre el «holismo local», que en cierta forma equivale a la estrategia wittgensteiniana del "parecido de familia", Kuhn propone un concepto de ciencia en donde los criterios son parte importante. Cuarto, y para redondear la respuesta al problema planteado, Kuhn propone que la racionalidad en la elección radica en que se ha de ser consecuente en la elección con los desiderata que se asumen, en caso contrario la elección puede ser calificada por los miembros de la comunidad científica como de «irracional».

La dicotomía normativo/descriptivo en el presente contexto puede formularse, siguiendo a Kuhn, en los siguiente términos: "isi se comprende suficientemente la diferencia entre describir la conducta, por una parte, y justificarla, por otra?»" ${ }^{21}$; es decir, no es suficiente, no tiene penetración filosófica, el hecho de mostrar que los científicos emplean de manera común y recurrentemente ciertos criterios, para de ahí concluir que el comportamiento

21 KUHN, T. S., "Racionalidad y elección de teorías", en ¿Qué son las revoluciones científicas?, Ediciones Altaya, S. A., Barcelona, 1994, p. 138. 
- pasado, presente y futuro- del científico queda justificado. En otras palabras, sostener la dicotomía es asumir que sólo hay dos formas de asumir una reflexión sobre la ciencia: la que defienden los partidarios del modelo racional del cambio científico, en donde los criterios de cientificidad se fijan de una vez por todas y son los que dictan cómo debe proceder el científico; la otra, consiste en describir las circunstancias, y de ser posible con los más mínimos detalles, en que se desenvuelve la actividad científica. Algunos filósofos están dispuestos a defender que el modo descriptivo da muy buenos frutos en la historia y la sociología de la ciencia - campos en los que por cierto Kuhn se desempeñó-, en tanto que el modo normativo sería característico de la reflexión filosófica.

La manera como Kuhn replantea la anterior pregunta es indicio de que no se acoge a tan venerada distinción, pues dice: "¿en qué circunstancias puede afirmarse con propiedad que ciertos criterios que se observa usar a los científicos al evaluar teorías son, de hecho, también bases racionales de sus juicios?» ${ }^{22}$. Ya en la Postscrip se oponía a la estrechez de la dicotomía; por tanto, vale la pena citarlo en extenso: "las páginas anteriores presentan un punto de vista o una teoría acerca de la naturaleza de la ciencia y, como otras filosofías de la ciencia, la teoría tiene consecuencias para el modo en que deben proceder los científicos si quieren que su empresa triunfe. Aunque no tiene que ser correcta, como ninguna otra teoría, sí aporta una base legítima para reiterados "debe ser» y "tiene que ser". A la inversa, un conjunto de razones para tomar en serio la teoría es que los científicos, cuyos métodos han sido desarrollados y seleccionados de acuerdo con su éxito, en realidad sí se comportan como la teoría dice que debe hacerlo. Mis generalizaciones descriptivas son prueba de la teoría precisamente, porque también pueden haberse derivado de ella, en tanto que, según otras opiniones de la naturaleza de la ciencia, constituyen un comportamiento anómalo... Creo yo que la circularidad de tal argumento no lo hace vicioso» ${ }^{23}$.

Kuhn no es el único filósofo que en la actualidad se opone a esta dicotomía ${ }^{24}$; por ejemplo, considero que la propuesta de Quine de naturalizar la

22 Ibid.

23 KUHN, T. S., La estructura de las revoluciones cientificas, p. 315 y 316 . Ver también KUHN, T. S., "Consideración en torno a mis críticos", p. 399.

24 Al respecto, ver: MOULINES, C. U, Pluralidad y Recursión. Estudios epistemológicos, Alianza Editorial, S. A., 1991, pp. 61-67 y pp. 76-80. De Quine, especialmente: QuINE, W. 
epistemología refuerza está tesis, al plantear que ciencia y filosofía forman un continuo y que, por tanto, "no hay filosofía primera", "no hay exilio cósmico". El rechazo de la dicotomía, como bien lo destaca Kuhn, no significa que el lado normativo de la filosofía haya desaparecido, puesto que, para decirlo con las palabras de Quine: la ciencia misma es la que nos lleva a decir, por ejemplo, que la única evidencia que hay para la ciencia es evidencia sensorial, de modo que no hay conocimiento extrasensorial. En síntesis, si hemos de llegar a una respuesta plausible sobre la racionalidad presente en la actividad científica, esta no puede encontrarse de manera enteramente a priori, desconociendo lo que de hecho se da en la práctica científica, sino que, por el contrario, es claro la importancia de acudir a los análisis de la conducta de los científicos para lograr una mejor comprensión y justificación, "fundamentación", de los procesos de elección de teoría que se suceden en el seno de las comunidades científicas.

El punto ahora es aproximar una noción de ciencia. Para esto, como ya se dijo, Kuhn recurre a la estrategia que Wittgenstein desarrolla en las Investigaciones filosóficas para aquellos conceptos cuya determinación no puede darse a través de definiciones o de una serie de condiciones necesarias y suficientes. Este tipo de procedimiento argumentativo cobró importancia en las últimas reflexiones de Kuhn, especialmente en lo que tiene que ver con su tesis «holismo local", la cual permite dar una mejor explicación de su tesis de la inconmensurabilidad. El holismo local plantea que los conceptos fundamentales de una teoría no pueden aprenderse o definirse de manera aislada, independientes unos de otros, sino que deben aprenderse en grupo. El ejemplo clásico es el de los conceptos de masa, fuerza y la segunda ley de la mecánica newtoniana.

En este contexto, Kuhn plantea que «identificar los referentes del vocabulario moderno de las disciplinas requiere acceso a un campo semántico que agrupa actividades con respecto a dimensiones como exactitud, belleza, poder predictivo, normatividad, generalidad, etcm ${ }^{25}$. En otras palabras, los criterios forman un campo semántico que permiten darle sentido al término

V., Del estímulo a la ciencia, Editorial Ariel, 1998, cap.II Naturalismo. GuerRERO, P., Recurriendo a la ciencia para comprender la ciencia. La epistemología naturalizada de Quine, Tesis, Pontificia Universidad Javeriana, Bogotá, 1998, cap. II Epistemología y semántica, pp. 48-55.

25 KuHN, T. S., "Racionalidad y elección de teorías», p. 148. 
moderno "ciencia". A través de las descripciones que proporciona este campo semántico es posible establecer ciertas distinciones entre disciplinas, lo cual permite calificar a algunas como científicas, a otras como no científicas y a otras como estando en el limbo. Es claro que el campo semántico no puede entenderse como una serie de criterios que estipulan las condiciones necesarias y suficientes para que una disciplina sea calificada como científica, simplemente resultan útiles para identificar disciplinas como ciencia. Con esto finaliza lo que podría llamarse la justificación plausible de Kuhn de los criterios de elección de teoría. Resta, entonces, abordar el de la racionalidad en la elección de teoría.

Este punto se teje con el anterior, pero para introducirlo más fácilmente vale la pena comenzar con una ilustración. Un individuo que pertenezca a una comunidad científica interesada en elegir entre dos teorías en competencia que, demos por caso, no difieren en más sino en su capacidad para resolver enigmas, y elige aún así, teniendo claro lo que las diferencia, aquella teoría con una menor capacidad para resolver problemas, desde luego que no hay lugar sino para que los miembros de la comunidad califiquen dicho proceder, bajo estas circunstancias, como no racional. Esto en lo que concierne al uso de los criterios, pero lo mismo vale para casos como el siguiente: digamos que el individuo anterior hace afirmaciones sinceras del siguiente tenor: «La ciencia $\mathrm{X}$ es menos exacta que la no-ciencia Y; por lo demás ambas ocupan la misma posición con respecto a todas las características". "Afirmaciones de esta clase ponen a la persona que las hace fuera de su comunidad lingüística. Persistir en ellas tiene como resultado la ruptura de la comunicación, y si se intenta justificar esta resistencia, se obtiene a menudo también como resultado acusaciones de irracionalismo" ${ }^{26}$.

La anterior situación, en los términos más teóricos del campo semántico del vocabulario disciplinar, podría expresarse del siguiente modo. Dentro de una comunidad lingüística es necesario respetar ciertas reglas semánticas y su abrogación por parte de uno de sus miembros simplemente indica: o bien

26 Ibid., p. 149. Esta tesis sobre la racionalidad ya estaba sugerida de alguna forma en "Notas sobre Lakatos", p. 523, cuando dice: "solo la persona que dice, por ejemplo: "la teoría A es más simple que la teoría $\mathrm{B}$, siendo iguales en todo lo demás; sin embargo, yo prefiero B", sólo una persona que toma decisiones estructuradas de este modo, viola lo que Lakatos llama el código de honor científico". 
que éste está optando por quedarse fuera del juego del lenguaje científico o bien que está rechazando el campo semántico mismo. En la primera situación es donde cabe el calificativo «irracional», en tanto que en la segunda es necesario que la persona presente alternativas al campo semántico dominante, lo cual debe resolverse a través de ajustar porciones amplias del vocabulario disciplinar; es decir, motivar un cambio en el concepto mismo de lo que es "racional» o «irracional" en la comunidad científica. En síntesis, uno, la racionalidad en la elección radica en que se ha de ser consecuente en la elección con los desiderata que se asumen, en caso contrario la elección puede calificarse, dentro de la comunidad científica, de «irracional» ${ }^{27}$; y, dos, la idea básica que se encuentra detrás de la anterior tesis es, para decirlo con los términos de Hoyningen-Huene, «que el problema de la racionalidad de la elección de teorías debe plantearse respecto a los grupos y no respecto a los individuos» ${ }^{28}$.

Finalmente, Kuhn concluye su análisis sobre la justificación en la elección racional de teoría, de manera un tanto enigmática y definitiva, así: "igual que "masa" y "fuerza", o "ciencia" y "arte", "racionalidad" y "justificación" son términos que se interdefinen. Un requisito para cualquiera de los dos es ajustarse a las limitaciones impuestas por la lógica. Otro requisito es ajustarse a las limitaciones impuestas por la experiencia en ausencia de buenas razones para lo contrario. Ambos requisitos ponen de manifiesto parte de lo que implica ser racional" 29 . Estas palabras contrastan de manera muy importante con aquellas que constituían la tesis fundamental en la época de La estructura: "la cuestión de la elección de una teoría no puede resolverse nunca de manera inequívoca sólo mediante la lógica y la experimentación» ${ }^{30}$.

27 NEWTON-SMITH defiende un concepto de racionalidad idéntico, pues dice: "La calificación de 'no racional', aplicada a este contexto —el de la actividad científica-, indica tan sólo que sus acciones no son racionales en relación a su finalidad en tanto científico. Sin embargo, no debe suponerse que la misma lleve implícita la idea de que el científico no actúa racionalmente. Dada su meta y sus creencias, muy bien puede ser ésa una estrategia racional para alcanzar la meta». NEWTON-SMITH, W. H., "La racionalidad científica", p. 21.

28 HOYNINGEN-HUENE, P., "Las interrelaciones entre la filosofía, la historia y la sociología de la ciencia en la teoría del desarrollo científicom, en Solıs, C. (Comp.), Alta tensión: Historia, filosofia y sociología de la ciencia. Ensayos en memoria de Thomas Kubn, Paidós, Barcelona, 1998, p. 104.

29 KuHN, T. S., "Racionalidad y elección de teorías", p. 151.

30 KuHN, T. S., La estructura de las revoluciones cientificas, p. 153. La cursiva es mía. 
Considero que la cuestión aquí tiene que ver con dónde se pone el acento, con establecer matices. En un comienzo Kuhn hace énfasis más en lo otro que en la lógica y la experiencia, y en sus escritos últimos hace afirmaciones que fuera de contexto parecen no distanciarse de las primeras, pero que vistas con mayor atención acentúan más la función de la lógica y la experiencia que la de las otras virtudes de una buena teoría ${ }^{31}$.

31 Para complementar: «supongamos ahora - plantea KUHN - que el propósito del científico al seleccionar teorías es llevar al máximo la eficacia en lo que he llamado en otro lugar la "resolución de enigmas». Desde este punto de vista, las teorías deben ser evaluadas utilizando consideraciones tales como su efectividad en proporcionar concordancias con los resultados del experimento y la observación», en KUHN, T. S., "Racionalidad y elección de teorías», pp. 138 y 139. 